\title{
Phenotype of Higher Post-load Insulin Response as a Predictor of All-cause Mortality and Cardiovascular Mortality in the Chinese Non- diabetes Population
}

\section{Xiaoxia Shen}

Chinese Academy of Medical Sciences \& Peking Union Medical College Fuwai Hospital https://orcid.org/0000-0001-6873-2310

\section{Siyao He}

Chinese Academy of Medical Sciences \& Peking Union Medical College Fuwai Hospital Jinping Wang

daqing first hospital

\section{Xin Qian}

Chinese Academy of Medical Sciences \& Peking Union Medical College Fuwai Hospital

\section{Hui Wang}

Chinese Academy of Medical Sciences \& Peking Union Medical College Fuwai Hospital

\section{Bo Zhang}

China-Japan Friendship Hospital

\section{Yanyan Chen}

Chinese Academy of Medical Sciences \& Peking Union Medical College Fuwai Hospital

Hui Li

Daqing Oilfield General Hospital

Guangwei Li ( $\sim$ guangwei_li45@126.com )

Center of Endocrinology, Fuwai Hospital, Chinese Academy of Medical Sciences

https://orcid.org/0000-0003-1061-7293

\section{Research}

Keywords: Insulin response, all-cause death, CVD death

Posted Date: September 9th, 2021

DOl: https://doi.org/10.21203/rs.3.rs-864005/v1

License: (c) (i) This work is licensed under a Creative Commons Attribution 4.0 International License. Read Full License 
Version of Record: A version of this preprint was published at Diabetology \& Metabolic Syndrome on January 28th, 2022. See the published version at https://doi.org/10.1186/s13098-022-00786-0. 


\section{Abstract}

Background This study aimed to assess whether a higher insulin response increased the long-term risk of mortality in a non-diabetes population.

Methods A total of 446 non-diabetes who participated in the Da Qing Diabetes Study were stratified into quartiles subgroups according to their baseline insulin area under the curve (AUC) during oral glucose tolerance test, defined as Q1, Q2, Q3 and Q4. The insulin AUC increased drastically from 75.23, 129.61, 179.06 to $309.85 \mathrm{mU} / \mathrm{L}$ across the four groups. The participants were followed from 1986 to 2016 to assess the risk of death in association with the magnitude of post-load insulin response.

Results The rates of all-cause death in participants who were assigned in the highest (Q4) and lowest (Q1) quartiles of insulin AUC groups were 17.58/1000 and 9.94/1000 person years during a 30-year period, respectively. Multiple Cox analyses with adjustment for baseline age, sex, smoking, post load glucose and other potential confounders showed that the risks of all-cause death in the Group 4 was significantly higher than Group 1(HR 1.90, 95\% Cl:1.12-3.23). The CVD death rate across these subgroups showed a similar trend (10.47/1000 person years in Group 4 vs 5.14/1000 person years in Group 1) as all-cause death, but it was not significantly different after the same adjustment.

Conclusions A higher post-load insulin response was significantly associated with a long-term increased risk of all-cause mortality in the Chinese non-diabetes population. Therefore, subjects featured by this phenotype was a potential important target for further intervention.

\section{Introduction}

Several studies had suggested that a higher blood insulin concentration in non-diabetes people may have contributed to the increased risk of death. However, the conclusion remained uncertain. The 22-year follow-up investigation of Helsinki Policemen Study showed that the highest quintile of the area under the insulin response curve among middle-aged men with normal blood glucose levels, compared with the combined 4 lower quintiles, was associated with a 2 -fold higher risk of stroke, but this association was not independent of other risk factors such as upper body obesity, raised blood pressure, and smoking [1]. In line with that result, an 11-year follow-up investigation of the Paris Prospective Study demonstrated that fasting plasma insulin level was an independent predictor of CVD death after adjusting for overt diabetes [2]. Interestingly, the results varied in the 15-year follow-up investigation of the same study, wherein the 2-h post-load plasma insulin level was a significant predictor of CVD-related death, while levels of blood glucose were not a significant predictor after the adjustment of plasma insulin levels. The author addressed that insulin response to the glucose challenge test requires further investigation as a potential risk factor for CAD and a potential target for further intervention [3].

A 30-year follow-up of The Daqing Diabetes Prevention Study showed that prevention or delay of diabetes can extend to the decrease of diabetes-related CVD events by $26 \%$ and a 1.44 -year longer life expectancy [4]. However, it is not clear whether the baseline high post-load insulin response is correlated 
with the risk of mortality in the non-diabetes population. The present analysis aimed to investigate whether the phenotype of a higher post-load insulin response in non-diabetes population predicted an increased long-term risk of CVD and all-cause mortality.

\section{Participants And Methods}

In total, 446 non-diabetes subjects, including 256 with impaired glucose tolerance (IGT) and 190 with normal glucose tolerance (NGT) identified by standard oral glucose tolerance test (OGTT) in 1986, were included in the present study. All the recruited participants had been part of the original Da Qing Diabetes Prevention Study. Details of the study design, methods, and population have been reported previously [57]. Briefly, in 1986, a total of 110,660 people (accounting for $50 \%$ of the adult residents) in Daqing, China, participated in a screening program for diabetes. Among them, 576 subjects with IGT and age- and sexmatched 519 subjects with NGT were identified based on the 1985 World Health Organization (WHO) criteria. In the present analysis,19 IGT subjects with FPG greater than $7.0 \mathrm{mml} / \mathrm{I}$ were excluded in order to follow the current diabetes criteria (WHO1999). Because main-focus of this study was the association of initial insulin response with the risk of death, only people with full data of plasma glucose, insulin levels at fasting and post-glucose load 1 hour and 2 hours, and outcome of death were recruited. The participants were stratified into four subgroups according to their baseline insulin AUC during a 75-g glucose load OGTT in 1986, defined as Q1, Q2, Q3 and Q4 groups; the insulin AUC across from Q1 to Q4 group of less than the 25th percentile $(75.2 \mathrm{mU} / \mathrm{L}), 25$ th -50 th $(129.6 \mathrm{mU} / \mathrm{L}), 50$ th -75 th $(179.1 \mathrm{mU} / \mathrm{L})$ and 75th percentile or more $(309.9 \mathrm{mU} / \mathrm{L})$ respectively. The insulin AUC during OGTT was calculated through trapezoidal estimation of the plasma insulin level at $0 \mathrm{~min}, 60 \mathrm{~min}$, and $120 \mathrm{~min}$ with the following formula: insulin $A U C=$ fasting insulin $(\mathrm{mU} / \mathrm{L}) / 2+$ insulin $1 \mathrm{~h}(\mathrm{mU} / \mathrm{L})+$ insulin $2 \mathrm{~h}(\mathrm{mU} / \mathrm{L}) / 2$. The date and cause of death were verified by review of patients' medical records, death certificates, or both. Details of follow-up and data collection were reported elsewhere [8].

\section{Outcomes}

Outcomes of the present study included all-cause mortality and CVD mortality.

\section{Ethics Approval}

The Human Ethics Committee of China-Japan Friendship Hospital and Fuwai Hospital approved the study, and written informed consent was obtained from all participants.

\section{Statistical Analysis}

Data are presented as mean $( \pm S D)$ or count (percentage). Descriptive statistics were used to compare groups of participants with different levels of insulin AUC. All-cause death and CVD death rates in the four quartiles of the insulin AUC groups were estimated as the number of deaths divided by the number of person-years. Confidence intervals (Cls) were calculated using Fisher's exact method. The incidences of all-cause and CVD mortality were compared among groups using Kaplan-Meier curves, and hazard 
ratios (HRs) were obtained using the Cox proportional hazards model. Two-tailed $\mathrm{P}$ values $<0.05$ were considered statistically significant. Multivariable models adjusted for age, sex, and other CVD risk factors (smoking, BMI, blood pressure, plasma total cholesterol, postprandial blood glucose and insulin AUC) were used to determine the influence of these factors on all-cause and CVD mortality rates over 30 years using Cox proportional hazards models and we used the multiple imputation method to impute the missing values for total serum cholesterol. Before multiple analysis, variance inflation factor (VIF) test was used to determine multicollinearity [9]. Only variables determined without multicollinearity (VIF values $<10$ ) were included in the model. Statistical analyses were performed using SAS for Windows, version 9.4 (SAS Institute Inc, Cary NC, USA).

\section{Results}

Across the four groups with increased levels of insulin AUC at baseline, the baseline fasting and postprandial plasma glucose, triglyceride, and cholesterol levels, blood pressure gradually increased. The insulin area under the curve increased drastically from $75.23,129.61,179.06$ to $309.85 \mathrm{mU} / \mathrm{L}$, with the BMI increasing from 22.83, 23.99, 25.63 to $27.29 \mathrm{~kg} / \mathrm{m}^{2}$. Males and smokers in Groups 3 and 4 was significantly lower than that in Groups 1 and 2 (Table 1). 
Table 1

Baseline characteristics of the study participants with different levels of insulin area under the curve (insulin-AUC) during oral glucose tolerance test

\begin{tabular}{|c|c|c|c|c|c|}
\hline & $\begin{array}{l}\text { Q1 } \\
(n=111)\end{array}$ & $\begin{array}{l}\text { Q2 } \\
(n=109)\end{array}$ & $\begin{array}{l}\text { Q3 } \\
(n=112)\end{array}$ & $\begin{array}{l}\text { Q4 } \\
(n=114)\end{array}$ & $P$ value \\
\hline Age (years) & $43.45 \pm 7.97$ & $42.71 \pm 8.94$ & $43.32 \pm 8.96$ & $45.77 \pm 8.18$ & 0.0390 \\
\hline Sex (\% men) & 54.05 & 59.63 & 47.32 & 41.23 & 0.0356 \\
\hline Smoking (\%) & 45.95 & 47.71 & 30.36 & 36.84 & 0.0276 \\
\hline $\mathrm{BMI}\left(\mathrm{kg} / \mathrm{m}^{2}\right)$ & $22.83 \pm 3.00$ & $23.99 \pm 3.07$ & $25.63 \pm 3.30$ & $27.29 \pm 3.81$ & $<0.0001$ \\
\hline FPG (mmol/L) & $4.92 \pm 0.71$ & $5.15 \pm 0.84$ & $5.21 \pm 0.72$ & $5.34 \pm 0.79$ & 0.0007 \\
\hline $2 \mathrm{hPG}(\mathrm{mmol} / \mathrm{L})$ & $6.19 \pm 2.04$ & $7.13 \pm 2.37$ & $7.50 \pm 2.03$ & $8.22 \pm 1.58$ & $<0.0001$ \\
\hline $\mathrm{SBP}(\mathrm{mmHg})$ & $121.9 \pm 20.3$ & $127.2 \pm 24.3$ & $130.8 \pm 20.0$ & $134.4 \pm 22.0$ & 0.0002 \\
\hline $\mathrm{DBP}(\mathrm{mmHg})$ & $81.38 \pm 12.3$ & $85.24 \pm 17.1$ & $87.10 \pm 12.3$ & $87.79 \pm 13.1$ & 0.0025 \\
\hline $\mathrm{CHO}(\mathrm{mmol} / \mathrm{L})$ & $4.88 \pm 1.34$ & $4.82 \pm 0.94$ & $5.10 \pm 1.16$ & $5.25 \pm 1.14$ & 0.0481 \\
\hline $\mathrm{TG}(\mathrm{mmol} / \mathrm{L})$ & $1.33 \pm 1.35$ & $1.19 \pm 0.62$ & $1.87 \pm 1.82$ & $2.12 \pm 2.01$ & 0.0004 \\
\hline FINS (mU/L) & $14.65 \pm 10.2$ & $15.46 \pm 7.1$ & $24.01 \pm 16.2$ & $33.02 \pm 19.0$ & $<0.0001$ \\
\hline 1hINS (mU/L) & $54.98 \pm 38.0$ & $85.88 \pm 17.9$ & $116.28 \pm 23.0$ & $199.38 \pm 69.5$ & $<0.0001$ \\
\hline 2hINS (mU/L) & $43.86 \pm 22.3$ & $71.99 \pm 32.4$ & $101.56 \pm 47.0$ & $187.93 \pm 79.0$ & $<0.0001$ \\
\hline Insulin-area (mU/L) & $75.23 \pm 19.4$ & $129.61 \pm 14.3$ & $179.06 \pm 16.4$ & $309.85 \pm 82.6$ & $<0.0001$ \\
\hline
\end{tabular}

During the 30-year follow-up period, all-cause death rates of the four groups were 9.94 (95\% Cl: 6.3213.56), 14.81 (95\% Cl: 10.27-19.34), 15.02 (95\% Cl: $10.48-19.57)$, and 17.58 (95\% Cl: 12.55-20.60) per 1000 person-years, respectively. The corresponding rates for CVD death were 5.14 (95\% Cl: 2.54-7.74), 6.50(95\% Cl: 3.50-9.50), 6.80 (95\% Cl: 3.74-9.85), and 10.47 (95\% Cl:6.59-14.35) (Table 2). 
Table 2

All-cause and CVD death rates in groups with different levels of baseline insulin-AUC over 30-year followup

$$
\text { Q1(n=111) Q2(n=109) Q3(n=112) Q4( } n=114)
$$

\section{All-cause death}

\begin{tabular}{|c|c|c|c|c|}
\hline Number & 29 & 41 & 42 & 47 \\
\hline Follow-up person-years & 2917 & 2769 & 2795 & 2674 \\
\hline \multirow{2}{*}{$\begin{array}{l}\text { Incidence per } 1000 \text { person years } \\
(95 \% \mathrm{Cl})\end{array}$} & 9.94 & 14.81 & 15.02 & 17.58 \\
\hline & $\begin{array}{l}(6.32- \\
13.56)\end{array}$ & $\begin{array}{l}(10.27- \\
19.34)\end{array}$ & $\begin{array}{l}(10.48- \\
19.57)\end{array}$ & $\begin{array}{l}(12.55- \\
22.60)\end{array}$ \\
\hline \multicolumn{5}{|l|}{ CVD deaths } \\
\hline Number & 15 & 18 & 19 & 28 \\
\hline Follow-up person-years & 2917 & 2769 & 2795 & 2674 \\
\hline \multirow{2}{*}{$\begin{array}{l}\text { Incidence per } 1000 \text { person years } \\
(95 \% \mathrm{Cl})\end{array}$} & 5.14 & 6.50 & 6.80 & 10.47 \\
\hline & $\begin{array}{l}(2.54- \\
7.74)\end{array}$ & $(3.50-9.50)$ & $(3.74-9.85)$ & $\begin{array}{l}(6.59- \\
14.35)\end{array}$ \\
\hline
\end{tabular}

Compared with Group 1, the risk of all-cause death was significantly higher in participants in Group 4 (HR $=2.14,95 \% \mathrm{Cl}: 1.34-3.42)$, Group $3(\mathrm{HR}=1.94,95 \% \mathrm{Cl}: 1.20-3.14)$, and Group 2 (HR=1.70, 95\% Cl: $1.06-$ 2.74). As for CVD death risk, participants in Group 4 showed a higher risk than those in Group 1 (HR $=$ $2.56,95 \% \mathrm{Cl}: 1.35-4.82)$ (Figure 1).

Multivariable analysis models which used to determine the effect of insulin AUC on all-cause and CVD mortality found after adjusting for age, sex, smoking, plasma total cholesterol, baseline BMI and systolic blood pressure (SBP), the risks of all-cause and CVD death in those within Group 4 were significantly higher than those within the Group 1 (HR 2.12 for all-cause and 2.09 for CVD deaths). Further adjusting for 2-h postprandial blood glucose, the risk of all-cause death in those with Group 4 remained significantly higher than those within the Group 1 (HR 1.90), while for CVD death in those with Group 4 was not significantly higher than those in the Group 1 (HR 1.77,95\% Cl:0.86-3.67) (Table 3). 
Table 3

Association between insulin-AUC at baseline and the risk of all-cause and CVD death over 30-year followup

\begin{tabular}{llll} 
Variable adjusted & Hazard Ratio & $95 \% \mathbf{C l}$ & $\mathbf{P}$ \\
\hline Model 1 for all-cause death & & & \\
Unadjusted & & & \\
\hline Q2/Q1 & 1.52 & $0.95-2.45$ & 0.0835 \\
\hline Q3/Q1 & 1.56 & $0.97-2.51$ & 0.0642 \\
\hline Q4/Q1 & 1.83 & $1.15-2.91$ & 0.0105 \\
\hline Age, sex adjusted & & & \\
\hline Q2/Q1 & 1.64 & $1.02-2.64$ & 0.0431 \\
\hline Q3/Q1 & 1.71 & $1.06-2.76$ & 0.0267 \\
\hline Q4/Q1 & 2.07 & $1.29-3.30$ & 0.0024 \\
\hline Age, sex, smoking adjusted & & & \\
\hline Q2/Q1 & 1.70 & $1.06-2.74$ & 0.0294 \\
\hline Q3/Q1 & 1.94 & $1.20-3.14$ & 0.0070 \\
\hline Q4/Q1 & 2.14 & $1.34-3.42$ & 0.0015
\end{tabular}

Age, sex, Smoking, cholesterol, BMI and SBP adjusted

$\begin{array}{llll}\text { Q2/Q1 } & 1.67 & 1.03-2.69 & 0.0374 \\ \text { Q3/Q1 } & 1.91 & 1.17-3.14 & 0.0101 \\ \text { Q4/Q1 } & 2.12 & 1.27-3.55 & 0.0043\end{array}$

Age, sex, Smoking, cholesterol, BMI, SBP, and 2hPG adjusted

$\begin{array}{llll}\text { Q2/Q1 } & 1.55 & 0.95-2.52 & 0.0767 \\ \text { Q3/Q1 } & 1.80 & 1.09-2.97 & 0.0212 \\ \text { Q4/Q1 } & 1.90 & 1.12-3.23 & 0.0166\end{array}$

\section{Model 2 for CVD death}

Unadjusted

Q2/Q1

1.30

$0.66-2.59$

0.4484

HR: Hazard ratio; Cl: Confidence interval; BMI: Body mass index; SBP: Systolic blood pressure; 2hPG: 2-hour plasma glucose; Q1, Q2, Q3, and Q4 were quantile groups of baseline insulin-AUC during 75g oral glucose tolerance test with the value of insulin-AUC of 75,129,179 and 309 respectively. 


\begin{tabular}{|llll|}
\hline Variable adjusted & Hazard Ratio & $95 \%$ Cl & P \\
\hline Q3/Q1 & 1.38 & $0.70-2.71$ & 0.3567 \\
\hline Q4/Q1 & 2.12 & $1.13-3.97$ & 0.0189 \\
\hline Age, sex adjusted & & & \\
\hline Q2/Q1 & 1.43 & $0.72-2.84$ & 0.3109 \\
\hline Q3/Q1 & 1.57 & $0.80-3.11$ & 0.1919 \\
\hline Q4/Q1 & 2.50 & $1.32-4.71$ & 0.0047 \\
\hline Age, sex, smoking adjusted & & & \\
\hline Q2/Q1 & 1.46 & $0.73-2.89$ & 0.2852 \\
\hline Q3/Q1 & 1.73 & $0.87-3.44$ & 0.1199 \\
\hline Q4/Q1 & 2.56 & $1.35-4.82$ & 0.0038 \\
\hline Age, sex, Smoking,cholesterol, BMI, and SBP adjusted & 1.38 & & \\
\hline Q2/Q1 & 1.52 & $0.69-2.75$ & 0.3642 \\
\hline Q3/Q1 & 2.09 & $0.75-3.11$ & 0.2471 \\
\hline Q4/Q1 & 1.44 & $1.03-4.22$ & 0.0401 \\
\hline Age, sex, Smoking, cholesterol, BMl, SBP and 2hPG adjusted & & \\
\hline Q2/Q1 & 1.27 & $0.63-2.56$ & 0.5048 \\
\hline Q3/Q1 & 1.77 & 0.3271 \\
\hline Q4/Q1 & & & 0.1222 \\
\hline $\begin{array}{l}\text { HR: Hazard ratio; Cl: Confidence interval; BMl: Body mass index; SBP: Systolic blood pressure; } 2 \mathrm{hPG:} \\
\text { 2-hour plasma glucose; Q1, Q2, Q3, and Q4 were quantile groups of baseline insulin-AUC during } 75 \mathrm{~g}\end{array}$ \\
\hline
\end{tabular}

\section{Discussion}

Many studies support the notion that CVD is related to insulin resistance [10-12]. However, results from different studies are controversial. In the 8.6 years' follow-up of the Prospective Study of Pravastatin in the Elderly (PROSPER), higher HOMA-IR was not associated with all-cause and CHD mortality or fatal/non-fatal CVD [13]. There is a paucity of evidence whether a higher post-load insulin response which induced by insulin resistance is a risk factor for CVD mortality in people with normal glucose status and impaired glucose tolerance.

In the present study, we found that non-diabetic Chinese people with higher insulin AUC in response to standard glucose challenge, an indicator of higher insulin demands for maintaining glucose metabolism, 
had significantly higher rates of all-cause and CVD mortality during the subsequent 30 years. Multiple regression analysis further confirmed that baseline insulin AUC significantly predicted these risks. A more interesting finding was that the predictive effect of insulin AUC on mortality was coexisted with a significant association between death and 2-hour post load glucose levels of the participants after controlling for the influence of traditional risk factors. It indicated that a higher post-load insulin response in this non-diabetes participants contributed to increased risk of mortality independent of other cardiovascular risk factors. This finding highlighted that a strategy only focusing on blood sugar control but ignoring role of the higher post-load insulin response may not reduce the overall risks of death. Therefore, to identify those non-diabetes subjects with higher post-load insulin response should be included in the strategies of lowing all-cause and CVD mortality. Accordingly, to eliminate these clustered risk factors, a package plan to deal with them in a coordinated and balanced manner is warrened.

Higher post-load insulin response was a manifestation of hyperinsulinemia. Why hyperinsulinemia increased the risk of death in the non-diabetes population? Clinically, an easily accepted reason is that the hyperinsulinemia in the non-diabetes subjects triggered and speed-up the onset of diabetes, and then the development to diabetes exacerbated the high risk of any death, especially CVD death. Findings in basic research also provided some insights into understanding the complexity between hyperinsulinemia and CVD risk. Chronic hyperinsulinemia causes coronary vasoconstriction by increasing the release of endothelin-1 and sympathetic nerve activity and by reducing endothelial response to vasodilators [14]. It is also associated with several dysregulations of coagulation and fibrinolysis [15]. Nolan et al proposed a more convincing concept of insulin mediated metabolic stress for conditions related to insulin resistance [16]. It was the insulin resistance had conferred protection against the nutrient overload and metabolic stress by limiting glucose flux into the cell. However, the iatrogenic hyperinsulinemia to override insulin resistance may also override the defence mechanism because the tissues will no longer gain protection from excess nutrient entry. Recently, this idea was supported by Stephan M's team, wherein they stated that too high systemic insulin levels are detrimental for body functions [17]. For non-diabetes, the higher post-load insulin response for maintaining normal blood glucose level may cause insulin-induced metabolic stress in target organs, thereby increasing the risk of death. This means that higher post-load insulin response is harmful to the body even in the non-diabetes population and should therefore be avoided. Most people with IGT and higher insulin response in the present study have deterioration of diabetes during the 30-year follow-up period. Therefore, findings in this non-diabetes population, especially both higher post load $2 \mathrm{~h}$ glucose and higher insulin response were significantly associated with the increased risk of all-cause death, may also have some implications for people with diabetes.

Type 2 diabetes with poor glycaemic control is often characterised by high blood glucose and low insulin levels and severe insulin resistance. The optimum and timely use of high-dose insulin response of the body may significantly improve the glucose control, but at the same time it had to pay the expense of hyperinsulinemia, which may lead to insulin-induced metabolic stress in the heart. It was reported that over-nutrition-induced metabolic stress is harmful to the heart (18-21). Like iatrogenic hyperinsulinemia, the higher post-load insulin response in patients with type 2 diabetes may lead to insulin-induced metabolic stress in the heart (metabolic cardiomyopathy), followed by increased risk of cardiac failure 
and arrhythmias (22-24). Unfortunately, hyperinsulinemia is almost inevitable for better glucose control because more than $80 \%$ of type 2 diabetes patients are resistant to insulin. Even worse, it is possible that this insulin resistance and hyperinsulinemia status may exist for lifetime. Thus, in the real-word clinical practice, how to balance the benefits of better blood glucose control and adverse effects of exogenic hyperinsulinemia in patients with type 2 diabetes has become a big challenge.

For alleviating higher insulin response, both lifestyle modification and using medications, such as GLP1 receptor agonists or metformin, should not be ignored. The present data showed that the baseline insulin AUC sequentially increased with the increase in BMI across the four groups, suggesting that insulininduced harm is more likely to occur in overweight/obese people. Therefore, losing weight and improving insulin sensitivity are good ways to avoid higher insulin response and prevent the development of diabetes.

Our study has several strengths. First, a standard OGTT was conducted at baseline; thus, plasma insulin AUC over 2 hours could be calculated. Second, very few people migrated away from the city, with a minimum rate of loss to follow-up. Third, the participants were followed up for 30 years, which was an optimal duration for patients to develop life-threatening complications such as CVD and stroke.

The limitations of the study should also be acknowledged. The sample size was relatively small. Furthermore, we had no systemic data of medications for the treatment of hypertension and hyperlipidaemia over the 30-year follow-up period; therefore, we are unable to evaluate the impact of these factors on the risk of mortality in the study population. High-quality research using real-world data may provide more information on this aspect.

In conclusion, together with an increase in post-load hyperglycaemia, a higher insulin response to glucose challenge significantly predicted the long-term risk of all-cause and CVD mortality in the Chinese nondiabetes population. Our findings suggest that identifying people with higher insulin response and taking appropriate measures to reduce an individual's insulin response to eliminate hyperinsulinemia may help in reducing all-cause and CVD mortality.

\section{Declarations}

\section{Availability of data and materials}

The datasets used during the current study are available from the corresponding author on reasonable request.

\section{Consent for publication}

Written and signed consents to publish the paper were obtained from the patients.

\section{Declaration of conflicting interests}


The authors declared no potential conflicts of interest with respect to the research, authorship, and/or publication of this article.

\section{Ethics statement}

The Human Ethics Committee of China-Japan Friendship Hospital and Fuwai Hospital approved the study, and written informed consent was obtained from all participants.

\section{Funding}

The author(s) disclosed receipt of the following financial support for the research, authorship, and/or publication of this article: the National Center for Chronic Disease Prevention and Health Promotion through CDC/WHO Cooperative Agreement No. U58/CCU424123-01-02 and the China-Japan Friendship Hospital.

\section{Authors' contributions}

$\mathrm{GL}, \mathrm{XS}, \mathrm{JW}, \mathrm{BZ}$ and $\mathrm{YC}$ conceived and designed the analysis. SH, XQ, JW, HW and HL collected the data. XS and GL performed the data analysis. XS and GL wrote and reviewed the manuscript. All authors have approved the final manuscript.

\section{Acknowledgements}

The authors thank all the participants who took part in the original Da Qing IGT and Diabetes Study, the Da Qing Diabetes Prevention Study, and the Da Qing Diabetes Prevention Follow-up Study. The authors thank Peter H. Bennett who provided advice about the manuscript. We also thank late Professor Xiaoren Pan, as this study would not have been possible without his leadership in the design and implementation of the original DQDPS.

\section{References}

1. Pyörälä $M$, Miettinen $H$, Laakso $M$, et al. Hyperinsulinemia and the risk of stroke in healthy middleaged men: the 22-year follow-up results of the Helsinki Policemen Study. Stroke. 1998; 29:1860-66. https://doi:10.1016/S2213-8587(14)70057-9

2. Eschwege E, Richard JL, Thibult N, et al. Coronary Heart Disease Mortality in Relation With Diabetes, Blood Glucose and Plasma Insulin Levels. The Paris Prospective Study, Ten Years Later. Horm Metab Res Suppl.1985; 15:41-46.

3. Fontbonne A1, Charles MA, Thibult N,et al. Hyperinsulinaemia as a predictor of coronary heart disease mortality in a healthy population: the Paris Prospective Study, 15-year follow-up. Diabetologia.1991; 34:356-361. https://doi:10.1007/BF 00405009

4. Gong Q, Zhang P, Wang J,et al .Morbidity and mortality after lifestyle intervention for people with impaired glucose tolerance: 30-year results of the Da Qing Diabetes Prevention Outcome Study. 
Lancet Diabetes Endocrinol.2019;7:452-461. https://doi:10.1016/S2213-8587(19)30093-2

5. Li G, Zhang P, Wang J, et al. The long-term effect of lifestyle interventions to prevent diabetes in the China Da Qing Diabetes Prevention Study: a 20-year follow-up study. Lancet.2008; 371:1783-1789. https://doi:10.1016/S0140-6736(08)60766-7

6. Hu YH, Pan XR, Liu PA, et al. Coronary heart disease and diabetic retinopathy in newly diagnosed diabetes in Da Qing, China: the Da Qing IGT and Diabetes Study. Acta Diabetol.1991; 28:169-173. https://doi:10.1007/BF 00579721

7. An Y, Zhang P, Wang J, et al. Cardiovascular and all-cause mortality over a 23-year period among Chinese with newly diagnosed diabetes in the Da Qing IGT and Diabetes Study. Diabetes Care.2015;38:1-7. https://doi:10.2337/dc14-2498

8. Gong Q, Zhang P, Wang J, et al. Changes in Mortality in People With IGT Before and After the Onset of Diabetes During the 23-Year Follow-up of the Da Qing Diabetes Prevention Study. Diabetes Care.2016; 39:1550-1555. https://doi:10.2337/dc16-0429

9. O’brien RM. A Caution Regarding Rules of Thumb for Variance Inflation Factors. Quality \& Quantity.2007; 41:673-690. https://10.1007/s11135-006-9018-6

10. Laakso M, Kuusisto J. Insulin resistance and hyperglycaemia in cardiovascular disease development. Nat Rev Endocrinol.2014;10:293-302. https://doi:10.1038/ nrendo. 2014.29

11. Ginsberg HN. Insulin resistance and cardiovascular disease. J Clin Investig.2000; 106:453-458. https://doi:10.1172/JCl10762

12. Bloomgarden ZT. Insulin resistance, dyslipidemia, and cardiovascular disease. Diabetes Care.2007; 30:2164-2170. https://doi:10.2337/dc07-zb08

13. Welsh P, Preiss D, Lloyd SM,et al. Contrasting associations of insulin resistance with diabetes, cardiovascular disease and all-cause mortality in the elderly: PROSPER long-term follow-up. Diabetologia.2014;57:2513-2520.https://doi:10.1007/s001 25-014-3383-9

14. Mahmoud AM, Szczurek MR, Blackburn BK. Hyperinsulinemia Augments endothelin-1 Protein Expression and Impairs Vasodilation of Human Skeletal Muscle Arterioles. Physiol Rep.2016;4: e12895. https://doi:10.14814/phy2.1289

15. Yudkin JS. Abnormalities of coagulation and fibrinolysis in insulin resistance. Evidence for a common antecedent? Diabetes Care.1999;22:C25-30

16. Nolan CJ, Ruderman NB, Kahn SE,et al. Insulin resistance as a physiological defense against metabolic stress: implications for the management of subsets of type 2 diabetes.

Diabetes.2015;64:673-86. https://doi:10.2337/db14-0694

17. Hubert Kolb, Kerstin Kempf, Martin Röhling,et al. Insulin: too much of a good thing is bad. BMC Med.2020;18:224. https://doi:10.1186/s12916-020-01688-6

18. Labbé SM, Grenier-Larouche T, Noll C, et al. Increased myocardial uptake of dietary fatty acids linked to cardiac dysfunction in glucose-intolerant humans. Diabetes.2012;61:2701-2710. https://doi:10.2337/db11-1805 
19. Stienstra R, Tack CJ, Kanneganti TD, et al. The inflammasome puts obesity in the danger zone. Cell Metab.2012;15:10-18. https://doi:10.1016/ j. cmet.2011.10.011

20. Fu Q, Xu B, Liu Y, et al. Insulin inhibits cardiac contractility by inducing a Gi-biased b2-adrenergic signaling in hearts. Diabetes.2014;63:2676-2689.https://doi: 10.2337/db13-1763

21. Tuomilehto J, Lindstrom J, Eriksson JG, et al. Prevention of type 2 diabetes mellitus by changes in lifestyle among subjects with impaired glucose tolerance. N Engl J Med.2001;344: 1343-50. https://doi:10.1056/NEJM200105033441801

22. Knowler WC, BarrettConnor E, Fowler SE, et al. Reduction in the incidence of type 2 diabetes with lifestyle intervention or metformin. N Engl J Med.2002;346: 393-403.

https://doi:10.1056/NEJMoa012512

23. Singh LP. The NLRP3 inflammasome and diabetic cardiomyopathy: editorial to: "Rosuvastatin alleviates diabetic cardiomyopathy by inhibiting NLRP3 inflammasome and MAPK pathways in a type 2 diabetes rat model" by Beibei Luo et al. Cardiovasc Drugs Ther.2014;28:5-6. https://doi:10.1007/s10557-013-6501-x

24. Jagasia D, Whiting JM, Concato J, et al. Effect of non-insulin-dependent diabetes mellitus on myocardial insulin responsiveness in patients with ischemic heart disease. Circulation.2001;103:1734-1739. https://doi:10.1161/01. cir.103.13.1734

25. Fullmer TM, Pei S, Zhu Y, et al. Insulin suppresses ischemic preconditioning-mediated cardio protection through Akt-dependent mechanisms. J Mol Cell Cardiol.2013;64:20-29. https://doi:10.1016/j.yjmcc.2013.08.005

\section{Figures}



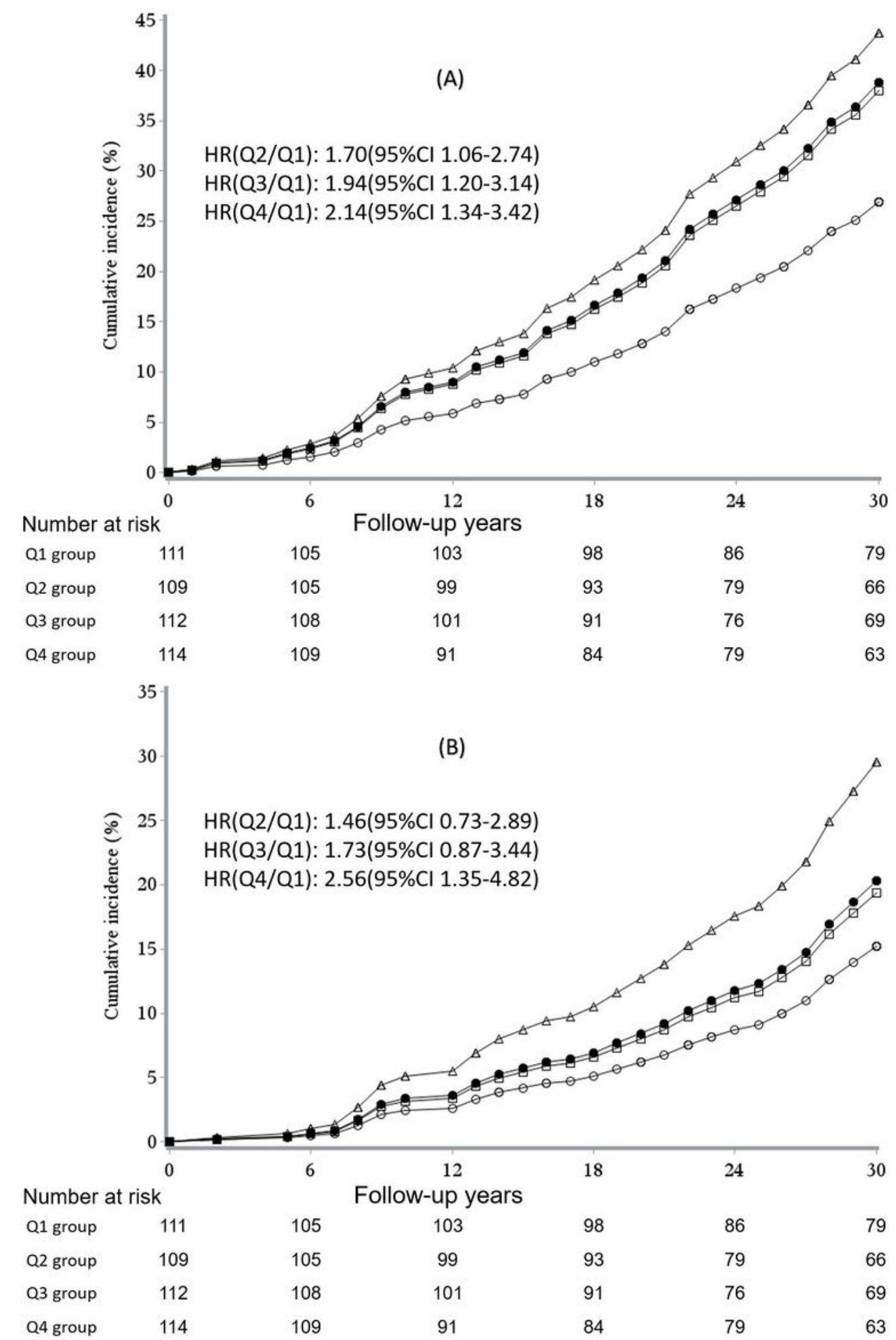

\section{Figure 1}

Cumulative incidence and hazard ratios (HRs) of all-cause and CVD death in 2016 among non-diabetes participants with different levels of insulin area under curve. A, Cumulative incidence of all-cause death. HRs was adjusted for age,sex,smoking. The triangles represent the top levels of insulin areas group(Q4), black circles represent the second level of insulin areas group(Q3), squares represent the third level of insulin areas group(Q2) and open circles represent the lowest level of insulin areas group(Q1). A, 
Cumulative incidence of CVD death. HRs was adjusted for age,sex,smoking. The triangles represent the top levels of insulin areas group(Q4), black circles represent the second level of insulin areas group(Q3), squares represent the third level of insulin areas group(Q2) and open circles represent the lowest level of insulin areas group(Q1). 